\title{
Resveratrol and/or Metformin Activates Serum Sirtuin-1 and Decreases Insulin Resistance in High Fructose-Fed Rats
}

\author{
Dalia Fathy El Agamy ${ }^{\mathrm{a}}$, Fatma El Desouky Ahmed ${ }^{\mathrm{b}}$
}

\begin{abstract}
a Department of medical Physiology, Faculty of Medicine, Menoufia University, Menoufia, Egypt. ${ }^{\mathrm{b}}$ department of Clinical Pharmacology, Faculty of Medicine, Menoufia University, Menoufia, Egypt.
\end{abstract}

Correspondence to: Dalia Fathy El Agamy, Department of medical Physiology, Faculty of Medicine, Menoufia University, Menoufia, Egypt

\section{Email:}

dalia.elagami@med.menoufia.edu.eg

Received:21 May 2020

Accepted: 9 June 2020

\begin{abstract}
:
Background: Insulin resistance is a risk factor for multiple diseases. Resveratrol is a potent activator of sirtuin 1 (SIRT1), which is implicated in the regulation of cellular functions and energy metabolism. Activation of SIRT1 is reported to increase insulin resistance. Aim: We aimed to consider the effects of individual or combined treatment with resveratrol and metformin on serum SIRT1 activity and insulin resistance in high fructose-fed rats. Methods: Forty male Wistar albino rats were divided into: (1) control group, (2) high fructose-fed group (HFF), (3) HFFresveratrol-treated group, (4) HFF-metformin-treated group, and (5) HFF with combined resveratrol and metformin-treated group. Body weight, fasting blood glucose and serum insulin were measured and HOMA-IR index was calculated. Lipid profile, inflammatory and oxidative stress markers and serum SIRT1 activity were also determined at the end of the study. Results: Individual treatment with resveratrol or metformin resulted in partial significant decrease
\end{abstract} in glucose and insulin levels and HOMA-IR index, which was associated with partial significant alteration in serum lipid profile, inflammatory and oxidative stress markers levels and significantly enhanced serum SIRT1 activity. However, combination therapy of both drugs resulted in maximal improvement in all measured biochemical parameters. Conclusion: Due to their synergistic action, the combination therapy of resveratrol and metformin significantly normalized serum SIRT1 activity and insulin resistance in high fructose-fed rats.

Key words: Fructose, Insulin resistance, Metformin, Resveratrol, Sirtuin. 



\section{Introduction}

Insulin resistance (IR) is a complex metabolic disorder and a risk factor for multiple diseases [1]. The dramatic increase in IR incidence can be attributed to lifestyle choices [2]. IR is the reduction of the insulin target cells, such as skeletal muscle, adipose tissue and liver, responses to the physiological concentration of insulin and impaired glucose metabolism [1].

IR leads to hyperglycemia, compensatory hyperinsulinemia and downregulation of glucose transporter type 4 (GLUT4) expression in the cell membrane [3]. Longterm high-sugar diet is a risk factor for the development for IR which contributes to deposition of plasma free fatty acids (FFAs) in non-fat cells, such as the skeletal muscle, liver, heart, and pancreas $[1,3,4]$.

Fructose is a natural sugar that is commonly used as a sweetener in beverages and processed foods. Elevated plasma levels of

insulin, free fatty acids, and triglycerides were shown in rats fed on high-fructose diet for 10 days [3] and the metabolism of excessive amount of fructose lead to development of fatty liver, IR and components of the metabolic syndrome [5]. Sirtuin is a member of the silent information regulator 2 family that was implicated as conserved regulators of lifespan [6].

There are seven sirtuins in mammals. Sirtuin 1 (SIRT1), a $\mathrm{NAD}^{+}$-dependent histone deacetylase, is the most characterized member that was found to be an important regulator of energy metabolism. SIRT1 is implicated in the regulation of several cellular functions such as glucose-lipid metabolism, mitochondrial biogenesis, inflammation, stress resistance, apoptosis, and autophagy [7].

Resveratrol, is a naturally occurring compound found in grapes, peanuts, and blueberries. It has strong antioxidant properties and had beneficial effects in wide range of diseases such as cardiovascular diseases, diabetes, inflammation, aging, and cancer [8].

Resveratrol is a potent SIRT1 activator [9, 10]. Metformin is the most widely-indicated insulin-sensitizing agent. It improves insulinmediated suppression of hepatic glucose production and enhances insulin-stimulated glucose disposal in skeletal muscle $[11,12]$. 
We aim to consider the effect of the SIRT1 activator, resveratrol, on serum SIRT1 activity and IR in high fructose-fed rats (HFF) and comparing its effects to metformin as well as to study the effect of combined treatment.

We also aim to demonstrate the possible associated changes in serum levels of lipid profile, tumor necrosis factor- $\alpha$ (TNF- $\alpha$ ), malondialdehyde (MDA), and superoxide dismutase (SOD) activity.

\section{Materials and Methods}

\section{Animals:}

This is a prospective study carried out on 40 male Wistar albino rats (150-200 g). The rats were kept in an animal house in Faculty of Medicine, Menoufia University with artificial light dark cycle of $12 \mathrm{~h}$ and at room temperature $26 \pm 1{ }^{\circ} \mathrm{C}$.

The rats were fed with standard rat chow and water adlibitum. Our study was approved by the ethical committee of the Faculty of Medicine, Menoufia University and the experiments were performed during the period from May 2019 to July 2019 in accordance with the ethical guidelines for the care and investigations in laboratory animals.

\section{Experimental Design and Collection of} Samples:

Rats were randomly divided into 5 equal groups ( $\mathrm{n}=8$ in each group). Control group: rats received $2 \mathrm{ml}$ distilled water daily by oral gavage, HFF group: rats were fed fructose rich diet $(60 \%$ fructose mixed with rats' chow) for 6 weeks [13], HFF resveratrol-treated group (HFF+RES): rats were fed fructose rich diet and resveratrol (Sigma-Aldrich Chemicals, St. Louis, MO) was freshly dissolved in $0.9 \%$ saline solution and administered in a dose of $20 \mathrm{mg} / \mathrm{Kg} /$ day for 6 weeks via oral gavage [14], HFF metformin-treated group (HFF+MET): rats were fed fructose rich diet and metformin (Actos-Lilly, Cairo, Egypt) was administered in a dose of $100 \mathrm{mg} / \mathrm{kg}$ body weight daily dissolved in $0.9 \%$ saline solution [15] by oral gavage daily for 6 weeks, and HFF with combined resveratrol and metformin-treated group $(\mathrm{HFF}+\mathrm{RES}+\mathrm{MET})$ : rats were fed fructose rich diet and received combined resveratrol and metformin treatment as described previously.

Body weight was determined at the end of the experiment. After treatment, animals were fasted overnight (10 PM - 10 AM) and blood glucose was measured using glucometer (Diavue, Muenster-Germany). Retro-orbital blood samples were then 
extracted via heparinized microcapillary tubes and left at room temperature for 30 minutes to clot. Samples were then centrifuged at $2000 \mathrm{rpm}$ for 10 minutes for separation of serum samples. Serum samples were stored at $-20{ }^{\circ} \mathrm{C}$ for further determination of fasting insulin, lipid profile, TNF- $\alpha$, MDA and SOD activity levels. Homeostatic model assessment (HOMA) index was calculated by the formula: [fasting blood insulin $(\mu \mathrm{U} / \mathrm{ml}) \times$ fasting blood glucose (mg/dl) / 405] [16].

Measurement of serum levels of MDA, SOD activity, triglycerides, cholesterol and lipoproteins:

Kits for enzymatic colorimetric determination of serum MDA (QuantiChrom ${ }^{\mathrm{TM}}$, BioAssay Systems, USA), SOD activity (EnzyChrom ${ }^{\mathrm{TM}}$, BioAssay Systems, USA), triglycerides, cholesterol and lipoproteins were obtained from Spainreact, Egypt. Their levels were determined using spectrophotometer (Shimadzu/Double beam spectro-photometer U.V.150, Germany) as mentioned in previous studies $[17,18]$.

Measurement of serum levels of insulin, TNF- $\alpha$, and SIRT1 activity:

Serum levels of insulin (DRG Instruments GmbH， Germany), TNF- $\alpha$ (Quantikine ${ }^{\circledR}$ ELISA, R\&D Systems Inc., MN, USA) and
SIRT1 activity (MyBioSource, Inc., USA) were tested using enzyme-linked immunosorbent technique using an automatic optical reader (SUNRISE Touchscreen, TECHAN, Salzburg, Austria) according to manufacturer's instructions.

\section{Statistical analysis:}

Results were expressed as mean \pm SD. SPSS software version 16.0 was used for performing all statistical analysis with applying Mann-Whitney $U$ test for testing significance. The probability value $<0.05$ was considered statistically significant.

\section{Results}

Insignificant change in body weight was observed when HFF group was compared to the control group and when the treated groups were compared to HFF and control groups $(\mathrm{P}>0.05)$. HFF+RES group showed insignificant change in all parameters when compared to the HFF+MET group ( $\mathrm{P}>0.05)$.

Fasting blood glucose, serum insulin, and HOMA-IR index:

Fasting blood glucose level was significantly higher in HFF group when compared to control group $(\mathrm{P}<0.001)$. HFF+RES and HFF+MET groups showed a significant decrease in fasting blood glucose to a level that was significantly lower than HFF group 
$(\mathrm{P}<0.001)$ and significantly higher than control $(\mathrm{P}=0.02$ and $\mathrm{P}=0.003$ respectively). HFF+RES+MET group showed significant decrease in blood glucose to a level that was significantly lower than HFF group $(\mathrm{P}<0.001)$ and insignificantly changed from control group $(\mathrm{P}=0.492)$ (Table 1$)$.

Serum insulin level was significantly increased in HFF group when compared to the control group $(\mathrm{P}<0.001)$. HFF+RES and HFF+MET groups showed significantly lower level of serum insulin when compared to HFF group $(\mathrm{P}<0.001)$. Serum insulin level in $\mathrm{HFF}+\mathrm{RES}$ and $\mathrm{HFF}+\mathrm{MET}$ groups was still significantly higher than control group $(\mathrm{P}=0.011$ and $\mathrm{P}=0.002$ respectively). HFF+RES+MET group showed significant decrease in serum insulin level when compared to $\mathrm{HFF}$ group $(\mathrm{P}<0.001)$ but it showed insignificant change when compared to the control group $(\mathrm{P}=0.512)$ (Table 1).

Insulin resistance was indicated by calculation of HOMA-IR index. There was significant increase in HOMA-IR index in HFF group, when compared to control group $(\mathrm{P}<0.001)$. HOMA-IR was significantly decreased in $\mathrm{HFF}+\mathrm{RES}$ and $\mathrm{HFF}+\mathrm{MET}$ groups to a level that was significantly lower when compared to HFF group $(\mathrm{P}<0.001)$ and significantly higher when compared to the control group $(\mathrm{P}=0.022$ and $\mathrm{P}<0.001$ respectively). HFF+RES+MET group showed significantly lower level of HOMAIR index when compared to HFF group $(\mathrm{P}<0.001)$ and insignificant change when compared to control group $(\mathrm{P}=0.503)$ (Table $1)$.

\section{SIRT1 activity level:}

Level of SIRT1 was significantly decreased in HFF group when compared to control group $(\mathrm{P}<0.001)$. HFF rats that received resveratrol or metformin showed significantly increased level of serum SIRT1 when compared to the HFF rats $(\mathrm{P}=0.005$ and $\mathrm{P}=0.031$ respectively). Serum SIRT1 level in $\mathrm{HFF}+\mathrm{RES}$ and $\mathrm{HFF}+\mathrm{MET}$ groups showed significantly lower level of SIRT1 when compared to the corresponding level in the control group $(\mathrm{P}=0.039$ and $\mathrm{P}=0.007$ respectively). HFF+RES+MER group showed significantly higher level of SIRT1 when compared to the corresponding level in HFF group $(\mathrm{P}<0.001)$ and insignificant change when compared to the corresponding level in control group ( $\mathrm{P}=0.324)$ (fig.1).

\section{Serum lipid profile:}

Serum cholesterol level was significantly increased in HFF group when compared to control group $(\mathrm{P}<0.001)$. Administration of resveratrol or metformin in $\mathrm{HFF}+\mathrm{RES}$ or $\mathrm{HFF}+\mathrm{MET}$ groups respectively significantly 
decreased serum cholesterol to a level that was significantly lower than HFF group $(\mathrm{P}=0.013$ and $\mathrm{P}=0.016$ respectively) and still significantly higher than control group $(\mathrm{P}=0.032$ and $\mathrm{P}=0.026$ respectively).

HFF+RES+MET group showed significantly lower level of cholesterol when compared to the corresponding level in HFF group $(\mathrm{P}<0.001)$ and insignificant change when compared to the corresponding level in control group ( $\mathrm{P}=0.446)$ (Table 2).

Serum triglycerides level was also significantly increased in HFF group when compared to control group $(\mathrm{P}<0.001)$.

$\mathrm{HFF}+\mathrm{RES}$ and $\mathrm{HFF}+\mathrm{MET}$ groups showed significantly decreased serum triglycerides level when compared to HFF group $(\mathrm{P}<0.001)$ and a significant change was observed when triglycerides levels in both groups were compared to the corresponding level in the control group $(\mathrm{P}=0.023$ and $\mathrm{P}=0.003$ respectively) (fig. 2 ).

HFF+RES+MET group showed significant decrease in triglycerides level when compared to HFF group $(\mathrm{P}<0.001)$ and insignificant change when compared to the corresponding level in control group $(\mathrm{P}=0.956)$ (Table 2).
There was significant increase in serum LDL in HFF group when compared to control group $(\mathrm{P}<0.001)$. HFF+RES and $\mathrm{HFF}+\mathrm{MET}$ groups showed significant decrease in serum LDL to a level that was significantly lower than HFF group $(\mathrm{P}<0.001$ and $\mathrm{P}=0.023$ respectively) and significantly higher than the control group $\quad(\mathrm{P}=0.03$ and $\quad \mathrm{P}<0.001$ respectively). HFF+RES+MET showed significantly lower level of LDL when compared to the corresponding value in HFF group $(\mathrm{P}<0.001)$ and insignificant change when compared to the corresponding value in control group ( $\mathrm{P}=0.994)$ (Table 2).

Serum HDL was significantly decreased in HFF group when compared to control group $(\mathrm{P}<0.001) . \mathrm{HFF}+\mathrm{RES}$ and $\mathrm{HFF}+\mathrm{MET}$ groups showed significantly higher level of serum HDL than the corresponding level in the HFF group $(\mathrm{P}=0.012$ and $\mathrm{P}=0.03$ respectively $)$.

Serum HDL in $\mathrm{HFF}+\mathrm{RES}$ or $\mathrm{HFF}+\mathrm{MET}$ group was still significantly lower when compared to the corresponding level in control group $(\mathrm{P}=0.031$ and $\mathrm{P}=0.013$ respectively). HFF+RES+MET group showed significantly higher level of HDL when compared to the corresponding level in the HFF group $(\mathrm{P}<0.001)$ and insignificant change when compared to the corresponding level in control group ( $\mathrm{P}=0.786)$ (Table 2). 

Serum level of TNF- $\alpha$, MDA, and SOD activity:

Serum TNF- $\alpha$ level was significantly increased in HFF group when compared to control group $(\mathrm{P}<0.001)$. Level of TNF- $\alpha$ was significantly decreased in HFF+RES and HFF+MET groups when compared to the corresponding level in HFF group $(\mathrm{P}<0.001)$.

Both groups also showed significant change when compared to the corresponding value in the control group $(\mathrm{P}<0.001)$.

HFF+RES+MET group showed significantly lower level of TNF- $\alpha$ when compared to the corresponding value in HFF group $(\mathrm{P}<0.001)$ and insignificant change when compared to the corresponding value in the control group ( $\mathrm{P}=0.12$ ) (fig. 1).

Serum MDA was significantly increased in HFF group when compared to control group $(\mathrm{P}<0.001) . \mathrm{HFF}+\mathrm{RES}$ and HFF+MET groups showed significantly lower level of serum MDA when compared to the HFF group ( $\mathrm{P}=0.025$ and $\mathrm{P}=0.037$ respectively). $\mathrm{A}$ significant change was also observed when serum MDA level in both groups was compared to the corresponding value in the control group $(\mathrm{P}=0.035$ and $\mathrm{P}=0.023$ respectively). $\mathrm{HFF}+\mathrm{RES}+\mathrm{MET}$ group showed significant decrease in MDA level when compared to the corresponding level in the HFF group $(\mathrm{P}<0.001)$ and insignificant change when compared to the corresponding value in the control group ( $\mathrm{P}=0.803)$ (fig. 2).

There was significant decrease in SOD activity level in HFF group when compared to control group $(\mathrm{P}<0.001)$. SOD activity level was significantly increased in $\mathrm{HFF}+\mathrm{RES}$ and HFF+MET groups when compared to HFF group $(\mathrm{P}<0.03$ and $\mathrm{P}=0.015$ respectively).

SOD activity levels in HFF+RES and $\mathrm{HFF}+\mathrm{MET}$ were significantly changed when compared to the corresponding levels in control group $(\mathrm{P}=0.009$ and $\mathrm{P}=0.019)$. HFF+RES+MET group showed significantly higher level of SOD activity when compared to the corresponding level in the HFF group $(\mathrm{P}<0.001)$ and insignificant change when compared to the corresponding value in the control group ( $\mathrm{P}=0.995)$ (fig. 2). 
Benha medical journal vol 37, issue 3, 2020

Table 1: Effect of resveratrol, metformin, and combined treatment on body weight $(\mathrm{g})$, blood glucose (mg/dl), fasting serum insulin $(\mu \mathrm{U} / \mathrm{ml})$ and HOMA-IR in HFF rats.

\begin{tabular}{lccccc}
\hline & Control & HFF & HFF+RES & HFF+MET & HFF+RES+MET \\
\hline Bogy Weight $(\mathbf{g})$ & $244 \pm 14$ & $256 \pm 14$ & $252 \pm 19$ & $255 \pm 11$ & $249 \pm 14$ \\
Blood Glucose $(\mathbf{m g} / \mathbf{d l})$ & $84.1 \pm 6.2$ & $120.8 \pm 14.1^{*}$ & $96.6 \pm 3.8^{* \#}$ & $99.6 \pm 3.8^{* \#}$ & $90.38 \pm 5.5^{\#}$ \\
Fasting Serum Insulin $(\boldsymbol{\mu U} / \mathbf{m l})$ & $14.3 \pm 2.2$ & $27.9 \pm 3.4^{*}$ & $19.6 \pm 2.8^{* \#}$ & $20.6 \pm 3.7^{* \#}$ & $16.8 \pm 3.5^{\#}$ \\
HOMA-IR & $3 \pm 0.6$ & $8.3 \pm 1.4^{*}$ & $4.7 \pm 0.6^{* \#}$ & $5.1 \pm 1^{* \#}$ & $3.7 \pm 0.8^{\#}$ \\
\hline
\end{tabular}

Data are expressed as mean $\pm \mathrm{SD}(\mathrm{n}=8)$. *: Significant as compared to the control group. \#: Significant as compared to the HFF group. \$: Significant as compared to the HFF+RES group. HFF: high fructose-fed rats; HFF+RES: high fructose-fed and resveratrol-treated group; HFF+MET: high fructose-fed and metformin-treated group; HFF+RES+MET: high fructose-fed with combined resveratrol and metformin-treated group; HOMA-IR: Homeostatic model assessment of insulin resistance.

.Table 2: Resveratrol, metformin, and combined treatment restores lipid profile in HFF rats

\begin{tabular}{llllll}
\hline & Control & HFF & HFF+RES & HFF+MET & HFF+RES+MET \\
\hline Serum Cholesterol (mg/dl) & $88 \pm 5$ & $124 \pm 9^{*}$ & $105 \pm 14^{* \#}$ & $106 \pm 12^{* \#}$ & $98 \pm 12^{\#}$ \\
Serum Triglycerides (mg/dl) & $60.6 \pm 2.5$ & $81.5 \pm 7.1^{*}$ & $68.3 \pm 4.4^{* \#}$ & $70.3 \pm 4.4^{* \#}$ & $62.3 \pm 4.4^{\#}$ \\
Serum LDL (mg/dl) & $46.75 \pm 6.9$ & $78.9 \pm 6.7^{*}$ & $53.25 \pm 8^{* \#}$ & $66.13 \pm 7.7^{* \#}$ & $48.3 \pm 7.7^{\#}$ \\
Serum HDL (mg/dl) & $44.5 \pm 6$ & $27 \pm 4.9^{*}$ & $36.25 \pm 5.4^{* \#}$ & $35.3 \pm 5^{* \#}$ & $41.45 \pm 5.4^{\#}$ \\
\hline
\end{tabular}

Data are expressed as mean \pm SD $(n=8)$. *: Significant as compared to the control group. \#: Significant as compared to the HFF group. \$: Significant as compared to the HFF+RES group. HFF: high fructose-fed rats; HFF+RES: high fructose-fed and resveratrol-treated group; HFF+MET: high fructose-fed and metformin-treated group; HFF+RES+MET: high fructose-fed with combined resveratrol and metformin-treated group; LDL: Low-density lipoprotein-cholesterol; HDL: High-density lipoprotein-cholesterol. 

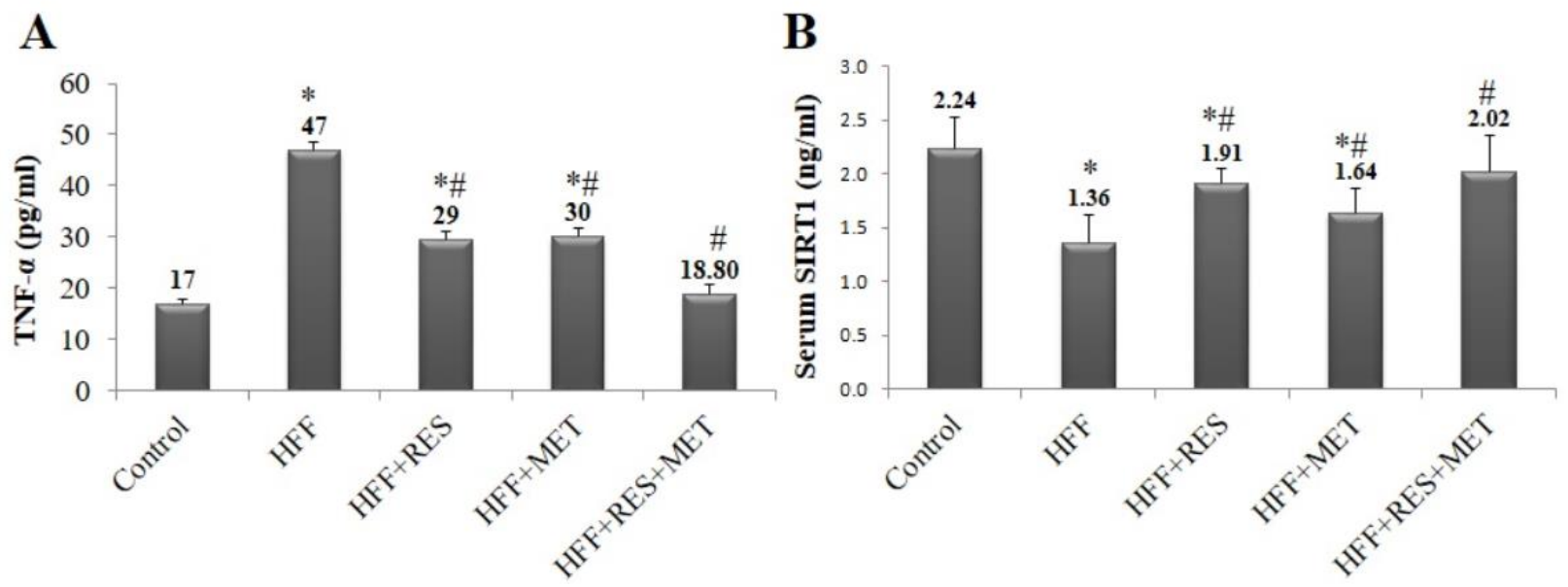

Figure 1. Resveratrol and/or metformin treatment restores serum (A) TNF- $\alpha$ and (B) SIRT1 activity in all studied groups. * Significant when compared to control group. ${ }^{\#}$ Significant when compared to HFF group. $(\mathrm{n}=8)$. TNF- $\alpha$ : tumor necrosis factor- $\alpha$, SIRT1: sirtuin-1, HFF: high fructose-fed rats; HFF+RES: high fructose-fed and resveratrol-treated group; HFF+MET: high fructose-fed and metformin-treated group; HFF+RES+MET: high fructosefed with combined resveratrol and metformin-treated group.
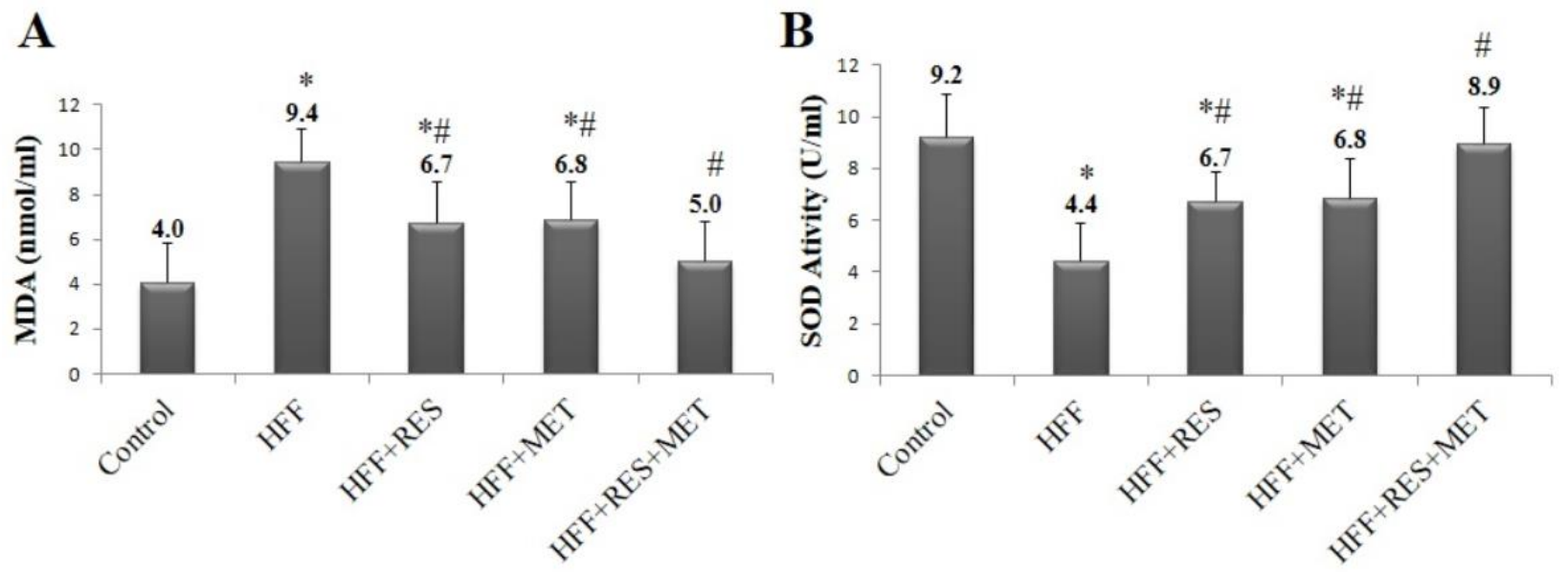

Figure 2. Resveratrol and/or metformin treatment improves serum oxidative status in all studied groups. * Significant when compared to control group. " Significant when compared to HFF group. ( $\mathrm{n}=8)$. MDA: malondialdehyde, SOD: superoxide dismutase, HFF: high fructose-fed rats; HFF+RES: high fructose-fed and resveratrol-treated group; HFF+MET: high fructose-fed and metformin-treated group; HFF+RES+MET: high fructosefed with combined resveratrol and metformin-treated group. 


\section{Discussion}

High fructose intake has a detrimental effect on insulin sensitivity. IR is a risk factor for multiple diseases such as micro- and macrovascular complications and development of cardiovascular disease and Type 2 diabetes [19, 20]. Activation of SIRT1 could improve IR and modulate glucose metabolism in insulin resistant conditions [21].

We investigated and compared the effects of individual or combined treatment with resveratrol, a potent SIRT1 activator, and metformin, an insulin sensitizer, on serum SIRT1 activity and IR in HFF rats and studied the associated changes in lipid profile, TNF- $\alpha$ and oxidative stress markers. Our study demonstrated that individual treatment with resveratrol or metformin partially enhanced serum SIRT1 activity, and partially improved IR and all measured biochemical parameters in HFF rats.

There was statistically insignificant difference in all studied parameters between HFF rats treated with resveratrol and those treated with metformin. Combined treatment with resveratrol and metformin had a more profound effect. We showed that combined treatment with resveratrol and metformin maximally improved SIRT1 activity and IR and maximally reversed the changes in serum lipid profile, serum TNF- $\alpha$, and oxidative stress markers in HFF rats.

Several studies have demonstrated the relationship between high fructose intake and the development of hyperglycemia and IR [2, 22].

Fructose has little influence on serum insulin concentration and plasma glucose levels in the short term. However, the negative longterm exposure to fructose leads to lipogenesis and greater elevation in triglycerides and cholesterol in an insulin independent manner, resulting in progressive fat deposition in skeletal muscle and accumulation of lipotoxic fat metabolites which in turn impair insulin signaling and induce IR mainly in liver and muscles [23]. IR and hyperinsulinemia are commonly associated with shift in cholesterol metabolism to increase synthesis and decrease absorption due to increase levels of triglycerides and FFAs [24, 25].

Sirtuins regulates a variety of cellular and biological processes. SIRT1 is the most extensively characterized Sirtuin. It is an epigenetic regulator that acts at chromatin 
level. SIRT1 has been shown to be attenuated in cells showing high IR such as heart, aortic blood vessels, and liver [26, 27]. And its activation was reported to increase insulin sensitivity [28, 29].

We observed significant decrease in serum SIRT1 activity level in HFF rats. However, Gok et al. [30] stated that serum SIRT1 protein level was significantly increased in patients with an insulin resistant state, type 2 diabetes mellitus (T2DM), and it has been reported to be a potentially new biomarker for T2DM.

However, the increased SIRT1 expression in patients with T2DM can be considered as a compensatory response in the body that may be overwhelmed. Previous studies have demonstrated that oxidative stress can directly or indirectly inhibit SIRT1 activity. Reactive oxygen species (ROS) production can be promoted due to ATP depletion, endoplasmic reticulum stress, uric acid formation, suppression of mitochondrial fatty acid oxidation, and deterioration of the antioxidant defense mechanisms in HFF rats $[31,32]$.

Other mechanisms that can induce oxidative stress in fructose overload is through hypertriglyceridemia and production of advanced glycation end products (AGEs) through fructose auto-oxidation that is ten times greater than those produced by glucose [33].Oxidative stress can induce oxidative modification of SIRT1 enzyme, decrease SIRT1 mRNA level, and control the activation of 5 ' adenosine monophosphateactivated protein kinase (AMPK) enzyme which can reciprocally activate SIRT1. Attenuation of SIRT1 enhances the NF-kB signaling which supports the inflammatory responses [34].

Induced TNF- $\alpha$ production was observed in acute and chronic hyperglycemic conditions [35]. Excess ROS is also implicated in impairment of insulin receptor signal transduction at various points, resulting in decreased GLUT4 expression in the cell membrane and IR in peripheral tissue [4].

Tumor NF- $\alpha$ is an important factor in the regulation of IR in HFF rats [36]. Excessive TNF- $\alpha$ production directly impairs glucose uptake and metabolism through modifications of insulin signal transduction [19] and is implicated in the generation of ROS and stimulation of hepatic lipogenesis $[37,38]$.

Individual treatment with insulin or resveratrol significantly increased SIRT1 activity and improved IR in HFF rats, and complete normalization was observed in 
HFF+RES+MET group. Resveratrol is a potent SIRT1 activator. Activation of SIRT1 directly affects insulin signaling in multiple insulin sensitive cells. It also decreases serum low-density lipoprotein (LDL) and total cholesterol and prevents accumulation of fatty acids [39].

SIRT1 activation protects cells from ROSinduced damage via inhibition of mitochondrial ROS generation and increasing NADPH levels [40]. SIRT1 can also enhance insulin signaling because of its antiinflammatory actions; it inhibits proinflammatory gene expression through deacetylation and inhibition of binding of nuclear factor kappa B to its target gene promoters [41].

Resveratrol can effectively reduce total cholesterol, triglycerides, and LDL levels in a dose effect manner, which may also be due to the phenolic hydroxyls contained in resveratrol [42]. It also acts as a free radical scavenger and upregulate endogenous cellular antioxidant system [43].

In agreement with us, Cheng et al. stated that resveratrol decreases ROS production, downregulates NADPH oxidase, and upregulates the level of SOD in rats with fructose-induced hypertension [44] due to activation of AMPK enzyme. Consistent with our results, Abd El-Haleim et al. [45] stated that resveratrol ameliorated TNF- $\alpha$ level in fructose-induced metabolic abnormalities in rats. Resveratrol exerts anti-inflammatory activities that mainly target cyclooxygenase (COX), 5-lipoxygenase and protein kinase B enzymes [46].

Metformin has a beneficial effect on reducing blood cholesterol and triglycerides in HFD rats [47]. Which explains the enhanced insulin sensitivity, significant decrease in fasting blood glucose and serum insulin levels and the significant increase in HOMAIR index in HFF+MET group. Metformin treatment was observed to enhance SIRT1 activity in $\mathrm{HFF}+\mathrm{MET}$ group; it induces AMPK activation that can function as a SIRT1 activator [48, 49].

In addition, consistent with our results, metformin attenuated oxidative stress in fructose-fed rats [50]. It enhances the plasma antioxidant capacity by increasing antioxidant enzymes activities (SOD and glutathione peroxidase) and decreasing the plasma MDA level in HFF rats [50]. We also observed that metformin partially attenuated TNF- $\alpha$ in HFF + MET rats. Metformin has several biologic activities including antiinflammatory and immunomodulatory properties [51]. Combined treatment with resveratrol and metformin completely 
normalized all studied parameters. This was in agreement with Frendo-Cumbo et al. [52] who reveals that the combination treatment with these compounds may have a greater efficacy in diet-induced IR.

\section{Conclusion}

Due to their synergistic action, the combination therapy of resveratrol and metformin significantly enhances serum SIRT1 activity and significantly alleviates IR in HFF rats via amelioration of hyperglycemic, hyperlipidemic, inflammatory and increased oxidative mechanisms.

\section{List of Abbreviations:}

AGEs: Advanced glycation end products

AMPK: Adenosine monophosphate-activated protein kinase

FFAs: Free fatty acids

GLUT4: Glucose transporter type 4

HDL: High-density lipoproteins

HFF: High fructose-fed

HOMA: Homeostatic model assessment

IR: Insulin resistance

LDL: Low density lipoproteins

MDA: Malondialdehyde

ROS: Reactive oxygen species

\section{SIRT1: Sirtuin 1}

SOD: Superoxide dismutase

T2DM: Type 2 diabetes mellitus

TNF- $\alpha$ : Tumor necrosis factor- $\alpha$

\section{References}

1. Yu J, Zheng J, Liu XF, Feng ZL, Zhang XP, Cao LL, et al.: Exercise improved lipid metabolism and insulin sensitivity in rats fed a high-fat diet by regulating glucose transporter 4 (GLUT4) and musclin expression. Braz J Med Biol Res. 2016; 49(5):e5129.

2. Baena M, Sanguesa G, Davalos A, Latasa M-J, Sala-Vila A, Sanchez RM, et al.: Fructose, but not glucose, impairs insulin signaling in the three major insulin-sensitive tissues. Sci. Rep. 2016; 6:26149.

3. Sah SP, Singh B, Choudhary S, Kumar A: Animal models of insulin resistance: A review. Pharmacol Rep. 2016; 68(6):1165-77.

4. Hurrle S, Hsu WH: The etiology of oxidative stress in insulin resistance. Biomed. J. 2017; 40(5):257-62

5. Priyadarshini E, Anuradha CV: Glucocorticoid Antagonism Reduces Insulin Resistance and Associated Lipid Abnormalities in HighFructose-Fed Mice. Can. J. Diabetes. 2017; 41(1):41-51.

6. Satoh A, Stein L, Imai S: The role of mammalian sirtuins in the regulation of metabolism, aging, and longevity. Handb. Exp. Pharmacol. 2011; 206:125-62. 
7. Cao Y, Jiang X, Ma H, Wang Y, Xue P, Liu Y: SIRT1 and insulin resistance. J Diabetes Complications. 2016; 30(1):178-83.

8. Kaur G, Padiya R, Adela R, Putcha UK, Reddy GS, Reddy BR, et al: Garlic and Resveratrol Attenuate Diabetic Complications, Loss of $\hat{\mathbf{I}}^{2}$ Cells, Pancreatic and Hepatic Oxidative Stress in Streptozotocin-Induced Diabetic Rats. Front pharmacol. 2016; 7:360.

9. Howitz KT, Bitterman KJ, Cohen HY, Lamming DW, Lavu S, Wood JG, et al.: Small molecule activators of sirtuins extend Saccharomyces cerevisiae lifespan. Nature. 2003; 425(6954):191-6.

10. Desai T, Koulajian K, Ivovic A, Breen DM, Luu L, Tsiani EL, et al.: Pharmacologic or genetic activation of SIRT1 attenuates the fat-induced decrease in beta-cell function in vivo. Nutr Diabetes. 2019; 9(1):11.

11. Giannarelli R, Aragona M, Coppelli A, Del Prato S: Reducing insulin resistance with metformin: the evidence today. Diabetes Metab. 2003; 29(4 Pt 2):6S28-6S35.

12. Miceli DD, Vidal PN, Batter MFC, Pignataro O, Castillo VA: Metformin reduces insulin resistance and the tendency toward hyperglycaemia and dyslipidaemia in dogs with hyperadrenocorticism. Open Vet J. 2018; 8(2):193-9.

13. Kitagawa A, Ohta Y, Ohashi K: Melatonin improves metabolic syndrome induced by high fructose intake in rats. J Pineal Res. 2012; 52(4):403-13.
14. Gaballah HH, Zakaria SS, Elbatsh MM, Tahoon NM: Modulatory effects of resveratrol on endoplasmic reticulum stress-associated apoptosis and oxido-inflammatory markers in a rat model of rotenone-induced Parkinson's disease. Chem Biol Interact. 2016; 251:10-6.

15. Choudhari VP, Gore KP, Pawar AT: Antidiabetic, antihyperlipidemic activities and herb-drug interaction of a polyherbal formulation in streptozotocin induced diabetic rats. $\mathrm{J}$ Ayurveda Integr Med. 2017; 8(4):218-25.

16. Matthews DR, Hosker JP, Rudenski AS, Naylor BA, Treacher DF, Turner RC: Homeostasis model assessment: insulin resistance and betacell function from fasting plasma glucose and insulin concentrations in man. Diabetologia. $1985 ; 28(7): 412-9$.

17. Satoh K: Serum lipid peroxide in cerebrovascular disorders determined by a new colorimetric method. Clin Chim Acta. 1978; 90(1):37-43.

18. Kuthan H, Haussmann HJ, Werringloer J: A spectrophotometric assay for superoxide dismutase activities in crude tissue fractions. Biochem J. 1986; 237(1):175-80.

19. Plomgaard P, Bouzakri K, Krogh-Madsen R, Mittendorfer B, Zierath JR, Pedersen BK: Tumor necrosis factor-alpha induces skeletal muscle insulin resistance in healthy human subjects via inhibition of Akt substrate 160 phosphorylation. Diabetes. 2005; 54(10):2939-45.

20. Weber KS, Simon M-C, Strassburger K, Markgraf DF, Buyken AE, Szendroedi J, et al.: Habitual Fructose Intake Relates to Insulin Sensitivity and Fatty Liver Index in Recent- 
Benha medical journal vol 37, issue 3, 2020

Onset Type 2 Diabetes Patients and Individuals without Diabetes. Nutrients. 2018; 10(6):774.

21. Liu P, Feng T, Zuo X, Wang X, Luo J, Li N, et al.: A novel SIRT1 activator E6155 improves insulin sensitivity in type 2 diabetic $\operatorname{KKA}(\mathrm{y})$ mice. Biochem. Biophys. Res. Commun. 2018; 498(3):633-9.

22. Malik VS, Popkin BM, Bray GA, Despres JP, Willett WC, Hu FB: Sugar-sweetened beverages and risk of metabolic syndrome and type 2 diabetes: a meta-analysis. Diabetes Care. 2010; 33(11):2477-83.

23. Samuel VT, Shulman GI: The pathogenesis of insulin resistance: integrating signaling pathways and substrate flux. J Clin Invest. 2016; 126(1):12-22.

24. Hoenig MR, Sellke FW: Insulin resistance is associated with increased cholesterol synthesis, decreased cholesterol absorption and enhanced lipid response to statin therapy. Atherosclerosis. 2010; 211(1):260-5.

25. Pihlajamaki J, Gylling H, Miettinen TA, Laakso M: Insulin resistance is associated with increased cholesterol synthesis and decreased cholesterol absorption in normoglycemic men. J Lipid Res. 2004; 45(3):507-12.

26. Lou P-H, Lucchinetti E, Scott KY, Huang Y, Gandhi M, Hersberger M, et al.: Alterations in fatty acid metabolism and sirtuin signaling characterize early type-2 diabetic hearts of fructose-fed rats. Physiol Rep. 2017; 5(16):e13388.

27. Pektaş MB, Sadi G, Akar F: Long-Term Dietary Fructose Causes Gender-Different Metabolic and
Vascular Dysfunction in Rats: Modulatory Effects of Resveratrol. Cell Physiol Biochem. 2015; 37(4):1407-20.

28. Reddy BR, Maitra S, Jhelum P, Kumar KP, Bagul PK, Kaur G, et al.: Sirtuin 1 and 7 mediate resveratrol-induced recovery from hyper-anxiety in high-fructose-fed prediabetic rats. J Biosci. 2016; 41(3):407-17.

29. Vila L, Roca C, Elias I, Casellas A, Lage R, Franckhauser S, et al.: AAV-mediated Sirt1 overexpression in skeletal muscle activates oxidative capacity but does not prevent insulin resistance. Mol Ther-Meth Clin D. 2016; $5: 16072$

30. Gok O, Karaali Z, Ergen A, Ekmekci SS, Abaci $\mathrm{N}$ : Serum sertuin 1 protein as a potential biomarker for type 2 diabetes: Increased expression of sirtuin 1 and the correlation with microRNAs. J Res Med Sci. 2019; 24:56.

31. Softic S, Cohen DE, Kahn CR: Role of Dietary Fructose and Hepatic De Novo Lipogenesis in Fatty Liver Disease. Digest Dis Sci. 2016; 61(5):1282-93.

32. Yilmaz Demirtas C, Bircan FS, Pasaoglu OT, Turkozkan N: The effects of resveratrol on hepatic oxidative stress in metabolic syndrome model induced by high fructose diet. Bratisl Lek Listy. 2018; 119(1):36-40.

33. Bernardes N, Ayyappan P, De Angelis K, Bagchi A, Akolkar G, da Silva Dias D, et al.: Excessive consumption of fructose causes cardiometabolic dysfunctions through oxidative stress and inflammation. Can J Physiol Pharmacol. 2017; 95(10):1078-90. 
34. Salminen A, Kaarniranta K, Kauppinen A: Crosstalk between Oxidative Stress and SIRT1: Impact on the Aging Process. Int J Mol Sci. 2013; 14(2):3834-59.

35. Esposito K, Nappo F, Marfella R, Giugliano G, Giugliano F, Ciotola M, et al.: Inflammatory cytokine concentrations are acutely increased by hyperglycemia in humans: role of oxidative stress. Circulation. 2002; 106(16):2067-72.

36. Togashi N, Ura N, Higashiura K, Murakami H, Shimamoto K: Effect of TNF-alpha--converting enzyme inhibitor on insulin resistance in fructose-fed rats. Hypertension. 2002; 39(2 Pt 2):578-80.

37. Sandoval R, Lazcano P, Ferrari F, Pinto-Pardo N, Gonzalez-Billault C, Utreras E: TNF- $\alpha$ Increases Production of Reactive Oxygen Species through Cdk5 Activation in Nociceptive Neurons. Front Physiol. 2018; 9:65.

38. Grunfeld C, Feingold KR: Tumor necrosis factor, cytokines, and the hyperlipidemia of infection. Trends Endocrinol Metab. 1991; 2(6):213-9.

39. Miranda MX, van Tits LJ, Lohmann C, Arsiwala T, Winnik S, Tailleux A, et al.: The Sirt1 activator SRT3025 provides atheroprotection in Apoe-/- mice by reducing hepatic Pcsk9 secretion and enhancing Ldlr expression. Eur Heart J. 2015; 36(1):51-9.

40. Santos L, Escande C, Denicola A: Potential Modulation of Sirtuins by Oxidative Stress. Oxid Med Cell Longev. 2016; 2016:9831825.

41. Stefanowicz M, NikoÅ,ajuk A, Matulewicz N, Karczewska-Kupczewska M: Adipose tissue, but not skeletal muscle, sirtuin 1 expression is decreased in obesity and related to insulin sensitivity. Endocrine. 2018; 60(2):263-71.

42. Xie H-c, Han H-P, Chen Z, He J-P: A study on the effect of resveratrol on lipid metabolism in hyperlipidemic mice. Afr J Tradit Complem. 2014; 11(1):209-12.

43. Hamza RZ, El-Shenawy NS: Anti-inflammatory and antioxidant role of resveratrol on nicotineinduced lung changes in male rats. Toxicol. Rep. 2017; 4:399-407.

44. Cheng PW, Ho WY, Su YT, Lu PJ, Chen BZ, Cheng WH, et al.: Resveratrol decreases fructose-induced oxidative stress, mediated by NADPH oxidase via an AMPK-dependent mechanism. $\mathrm{Br} \quad \mathrm{J}$ Pharmacol. 2014; 171(11):2739-50.

45. Abd El-Haleim EA, Bahgat AK, Saleh S: Resveratrol and fenofibrate ameliorate fructoseinduced nonalcoholic steatohepatitis by modulation of genes expression. World $\mathrm{J}$ Gastroenterol. 2016; 22(10):2931-48.

46. Salehi B, Mishra AP, Nigam M, Sener B, Kilic M, Sharifi-Rad M, et al.: Resveratrol: A DoubleEdged Sword in Health Benefits. Biomedicines. 2018; 6(3):91.

47. Ali M, Al-Qattan KK, Al-Enezi F, Khanafer RM, Mustafa T: Effect of allicin from garlic powder on serum lipids and blood pressure in rats fed with a high cholesterol diet. Prostaglandins Leukot Essent Fatty Acids. 2000; 62(4):253-9.

48. Ruderman NB, Xu XJ, Nelson L, Cacicedo JM, Saha AK, Lan F, et al.: AMPK and SIRT1: a long-standing partnership? Am J Physiol-Endoc M. 2010; 298(4):E751-E60 
49. Karise I, Ornellas F, Barbosa-da-Silva S, Matsuura C, del Sol M, Aguila MB, et al.: Liver and Metformin: Lessons of a fructose diet in mice. Biochim. Open. 2017; 4:19-30.

50. Vazquez-Prieto MA, Rodriguez Lanzi C, Lembo C, Galmarini CR, Miatello RM: Garlic and onion attenuates vascular inflammation and oxidative stress in fructose-fed rats. J Nutr Metab. 2011; 2011:475216.

51. Shin J-H, Ryu JH, Kang MJ, Hwang CR, Han J, Kang D: Short-term heating reduces the anti- inflammatory effects of fresh raw garlic extracts on the LPS-induced production of NO and proinflammatory cytokines by downregulating allicin activity in RAW 264.7 macrophages. Food Chem Toxicol. 2013; 58:545-51.

52. Frendo-Cumbo S, MacPherson RE, Wright DC. Beneficial effects of combined resveratrol and metformin therapy in treating diet-induced insulin resistance. Physiol. Rep. 2016 4(15):e12877.

To cite this article: Dalia Fathy El Agamy, Fatma El Desouky Ahmed. Resveratrol and/or Metformin Activates Serum Sirtuin-1 and Decreases Insulin Resistance in High Fructose-Fed Rats. BMFJ 2020; 37(3): 561-577, DOI: 10.21608/bmfj.2020.30792.1268 